Poincare Journal of Analysis 83 Applications

Vol. 2018 (2(II)), Special Issue (IWWFA-III, Delhi), 1-11

(C) Poincare Publishers

\title{
$J$-fusion frame and its application in Krein spaces
}

\author{
SHIBASHIS KARMAKAR
}

\author{
Date of Receiving : $\quad$ : 07.01 .2018 \\ Date of Revision : $\quad 15.07 .2018$ \\ Date of Acceptance : 02.08 .2018
}

\begin{abstract}
In this article we introduce the notion of $J$-fusion frame for a Krein space $\mathbb{K}$. We prove some basic results regarding $J$-fusion frame. We also relate this concept with fusion frame for Hilbert spaces and also with $J$-frame for Krein spaces. Further, we prove that $J$-fusion frame is actually a generalization of $J$-frames and we also explore some basic properties of $J$-fusion frame operator.
\end{abstract}

\section{Introduction}

The frame theory was introduced by Duffin and Schaeffer [1] in the year 1952 . But after the work of Daubechies et al. [2], frame theory developed rapidly. Now a days frame theory has major applications in signal processing, image processing, data compression, sampling theory and many more. One of the most important applications of frame theory is to calculate the effect of losses in packet-based communication system and in data transmission. In order to tackle this problem fusion frame theory was introduced. The idea behind fusion frame is to construct local frames and add them together to get the global frame. Fornasier [8] used this idea to quasi-orthogonal subspaces. Casazza et al. [5] formulated a general method to introduce fusion frame in Hilbert spaces.

Since fusion frame in Hilbert space has such huge applications, so it is a natural demand to extend these ideas in Banach space frame theory and also in Krein space frame theory. Some works had already been done in this direction $[9,10]$. In this article we are interested to extend the idea of fusion frame in Krein spaces. Krein space has some interesting applications in modern analysis. The theory of frame for Krein spaces can be found in $[11,12,13,15,16]$. Acosta-Humánez et al. [14] first defined fusion

2010 Mathematics Subject Classification. 42C15, 46C05, 46C20.

Key words and phrases. Krein Space, fusion frame, $J$-frame, $J$-orthogonal projection, uniformly $J$-definite subspace.

The author gratefully appreciate the guidance of Dr. Kallol Paul, Professor of Mathematics, Jadavpur University and Dr. Sk. Monowar Hossein, Professor of Mathematics \& Statistics, Aliah University for preparing this manuscript. The author also acknowledge Balagarh Bijoy Krishna Mahavidyalaya, West Bengal, for providing the necessary support.

Communicated by: Chander Shekhar 